\title{
The impact of pediatric emergency department crowding on patient and health care system outcomes: a multicentre cohort study
}

\author{
Quynh Doan MDCM PhD, Hubert Wong PhD, Garth Meckler MD MSHS, David Johnson MD, \\ Antonia Stang MDCM MSc, Andrew Dixon MD, Scott Sawyer MD, Tania Principi MD MSc, April J. Kam MD MScPH, \\ Gary Joubert MD, Jocelyn Gravel MD MSc, Mona Jabbour MD MEd, Astrid Guttmann MDCM MSc; for Pediatric \\ Emergency Research Canada (PERC)
}

Cite as: CMAJ 2019 June 10;191:E627-35. doi: 10.1503/cmaj.181426

See related article at www.cmaj.ca/lookup/doi/10.1503/cmaj.190610

\begin{abstract}
BACKGROUND: Emergency department overcrowding has been associated with increased odds of hospital admission and mortality after discharge from the emergency department in predominantly adult cohorts. The objective of this study was to evaluate the association between crowding and the odds of several adverse outcomes among children seen at a pediatric emergency department.
\end{abstract}

METHODS: We conducted a retrospective cohort study involving all children visiting 8 Canadian pediatric emergency departments across 4 provinces between 2010 and 2014. We analyzed the association between mean departmental length of stay for each index visit and hospital admission within 7 days or death within
14 days of emergency department discharge, as well as hospital admission at index visit and return visits within 7 days, using mixed-effects logistic regression modelling.

RESULTS: A total of 1931465 index visits occurred across study sites over the 5-year period, with little variation in index visit hospital admission or median length of stay. Hospital admission within 7 days of discharge and 14-day mortality were low across provinces $(0.8 \%-1.5 \%$ and $<10$ per 100000 visits, respectively), and their association with mean departmental length of stay varied by triage categories and across sites but was not significant. There were increased odds of hospital admission at the index visit with increasing departmental crowding among visits triaged to Canadian Triage and Acuity Scale (CTAS) score 1-2 (odds ratios [ORs] ranged from 1.01 to 1.08 ) and return visits among patients with a CTAS score of 4-5 discharged at the index visit at some sites (ORs ranged from 1.00 to 1.06 ).

INTERPRETATION: Emergency department crowding was not significantly associated with hospital admission within 7 days of the emergency department visit or mortality in children. However, it was associated with increased hospital admission at the index visit for the sickest children, and with return visits to the emergency department for those less sick.
E mergency department overcrowding is a complex systems problem and an important health services and public health concern. Although the magnitude of emergency department overcrowding varies regionally, the Canadian Association of Emergency Physicians has identified overcrowding as a serious problem across Canada, particularly at trauma and tertiary care centres. ${ }^{1}$ In North America, emergency department overcrowding has been associated with reduced quality of care, negative clinical outcomes (mortality and morbidity), and decreased patient and physician satisfaction. ${ }^{2-8}$ Some studies suggest an increased risk of in-patient mortality, prolonged hospital admissions and increased costs associated with increased emergency department volume. ${ }^{9} \mathrm{~A}$ population-based Ontario study found that emergency department visits during shifts with a longer departmental mean length of stay were associated with higher odds of hospital admission and mortality within 7 days of discharge from the emergency department. ${ }^{10}$

Although delay in transfer of admitted patients (i.e., "boarded" patients) in the emergency department ${ }^{6}$ is the 
primary cause of overcrowding in general emergency departments, increasing visit volumes and operational inefficiencies appear to drive overcrowding in pediatric emergency departments. ${ }^{11,12}$ Studies of overcrowding in pediatric emergency departments have identified important effects on quality of care, including delays in timely use of pain assessment scores for injuries, antibiotic administration for febrile neonates, analgesia for sickle cell crises or long bone fracture, and timely treatment of asthma. ${ }^{13-15}$ Evidence supporting an association between measures of crowding in the pediatric emergency department and patient outcomes or impact on health care use, however, is lacking.

This study explores the association between crowding in the pediatric emergency department at the time of patient arrival and hospital admission within 7 days or death within 14 days of discharge from an index visit, as well as rates of hospital admission at index visit and return visits to the emergency department within 7 days of emergency department discharge across 8 pediatric emergency departments in Canada.

\section{Methods}

\section{Study design, population and setting}

We conducted a retrospective cohort study of all patient visits to 8 Canadian pediatric emergency departments from Jan. 1, 2010, to Dec. 31, 2014, inclusive. All study sites are tertiary care pediatric emergency departments (study sites are listed in Appendix 1, available at www.cmaj.ca/lookup/suppl/doi:10.1503/cmaj .181426/-/DC1) in 4 provinces (British Columbia, Alberta, Manitoba and Ontario) that treat an average of 30000-60000 children annually. We included all emergency department visits of patients younger than 18 years, without exclusion. Of note, data for pediatric emergency departments in Ontario included only visits associated with an Ontario Health Insurance Plan number. We obtained de-identified visit-level data (visitors from abroad or surrounding provinces were not captured).

We defined an index visit as one with no prior visit within 7 days. For each index visit, we obtained demographic information (age in years and sex), visit characteristics (acuity level categorized by the pediatric Canadian Triage and Acuity Scale [CTAS], ${ }^{16}$ date and time of arrival), emergency department disposition (admitted or discharged), and outcomes after emergency department discharge (return visits to the emergency department, hospital admission and death).

\section{Exposure variable}

Our measure of crowding in the pediatric emergency department was the mean departmental length of stay, defined for each index visit to the pediatric emergency department as the mean length of stay for all patients who arrived at the pediatric emergency department within 8 hours before the index patient's arrival, excluding those who left without being seen. Length of stay was defined as the period from earliest recorded arrival time to the pediatric emergency department (registration or triage based on each site's recording practice) until departure. Although many factors contribute to overcrowding, length of stay is commonly used as a measure of crowding because it reflects the combined results of volume surges, increased resource demands and throughput delays. ${ }^{10,11,17-19}$

\section{Outcome measures}

The primary measure of adverse outcomes was either hospital admission within 7 days to one of the study sites or death within 14 days of an index visit among those discharged home or who left without being seen. Secondary measures of adverse outcomes included (a) hospital admission at the index visit and (b) return visits to the emergency department (scheduled or unscheduled) within 7 days of the index visit if the patient was discharged or left without being seen. We also reported measures of use at each study site over the 5-year study period (total volumes stratified by acuity level). All outcomes were evaluated by triage acuity, grouped as high (CTAS score 1-2), medium (CTAS score 3 ) and low (CTAS score 4-5). The CTAS is a validated scale used for triage in emergency departments across Canada to stratify patient acuity, risk and care needs based on presenting signs and symptoms. ${ }^{15}$ Categories of the CTAS include 1 (resuscitation; e.g., cardiac arrest), 2 (emergent; e.g., severe asthma), 3 (urgent; e.g., abdominal pain), 4 (less urgent; e.g., upper respiratory tract infection) and 5 (nonurgent; e.g., benign rash).

\section{Data sources}

Province-level administrative databases were available for 3 (Alberta, Manitoba and Ontario) of the 4 provinces, which allowed us to capture outcomes within the regions even if they did not occur at the original site. In BC, data were limited to the single pediatric emergency department, thus return visits to the emergency department, hospital admission and death at another site within the region were not captured. Data differed among provinces with regard to mortality: in Ontario, complete vital statistics data (death anywhere, not just in hospital) were used, whereas only in-hospital mortality data were available from the other 3 provinces. Further details regarding data sources are available in Appendix 2, available at www.cmaj.ca/ lookup/suppl/doi:10.1503/cmaj.181426/-/DC1.

\section{Statistical analysis}

We used descriptive statistics to summarize visit characteristics and health care use by province. We used mixed-effects logistic regression to test the association between departmental length of stay before arrival and our outcomes for patients with low-, medium- and high-acuity visits. Models treated patients arriving during the same shift in each pediatric emergency department as a cluster and included a random intercept for each cluster. Based on empirical observations (spike in event rates in the group aged < $1 \mathrm{yr}$ ), a nonlinear adjustment for age was included in the models. For each study hospital, we analyzed the odds of hospital admission within 7 days or death within 14 days of discharge from the pediatric emergency department, odds and 95\% confidence intervals (Cls) of hospital admission at the index visit, and odds of return visits to the emergency department within 7 days of discharge from the pediatric emergency 
department, with each hour increase in mean departmental length of stay before the index visit's arrival. For Alberta and Ontario, we pooled results from the multiple sites within each province using weights proportioned to the number of visits to obtain summary results for each province. Similarly, analyses combining provinces used weights proportional to the number of visits analyzed in each province. Age, sex, acuity (CTAS score), shift of arrival (day, evening or night) and season of arrival were included as potential confounders. The inclusion of season, however, led to problems with convergence during model fitting, and in cases where convergence was not a problem, it did not change the results. Hence, we excluded season in the final models. Only available data for each variable were analyzed. Given the large pool of data, and the low rate of missing data, we did not conduct sensitivity analyses. We did not adjust for multiple comparisons.

\section{Ethics approval}

Research ethics board reviews were conducted, and approvals were obtained for all participating sites.

Of note, because $B C$ and Manitoba have only 1 pediatric emergency department each, data from these sites were identifiable. We engaged pediatric emergency department leadership from both sites, and neither had reservations about publishing the results.

Table 1: Distribution of departmental length of stay during 8-hour periods before arrival index within and across provinces (2010-2014)

\begin{tabular}{|c|c|c|}
\hline \multirow[b]{2}{*}{ Province } & \multicolumn{2}{|c|}{ Length of stay, hr } \\
\hline & Mean \pm SD & Median (IQR) \\
\hline Ontario & $3.6 \pm 1.1$ & $3.5(2.9-4.2)$ \\
\hline Manitoba & $2.9 \pm 1.3$ & $2.8(2.3-3.4)$ \\
\hline Alberta & $3.4 \pm 0.9$ & $3.2(2.7-3.9)$ \\
\hline British Columbia & $3.5 \pm 0.9$ & $3.5(2.9-4.1)$ \\
\hline
\end{tabular}

\section{Results}

During the study period, a total of 1931465 index visits occurred across study sites. The 4 provinces had an annual average of 386293 index visits to pediatric emergency departments, and almost $90 \%$ were triaged as CTAS 3 or 4 (equally divided). We noted missing data in less than $2 \%$ of visits in each province. We observed modest variations in the proportion of visits resulting in hospital admission or the patient leaving without being seen at the index visit, with no clear pattern in median length of stay. Details by province and year are reported in Appendix 3, available at www.cmaj.ca/lookup/ suppl/doi:10.1503/cmaj.181426/-/DC1. The mean departmental length of stay varied both across and within provinces (Table 1 ). Across provinces, the mean length of stay ranged from 2.9 hours to 3.6 hours; within provinces, standard deviations ranged from 0.9 to 1.3 hours.

Overall, the prevalence of adverse outcomes was low: $0.8 \%$ to $1.5 \%$ of index visits to the emergency department resulted in hospital admission within 7 days of emergency department discharge. Mortality within 14 days of emergency department discharge was rare at less than 10 per 100000 visits to the pediatric emergency department (Table 2). Figure 1 depicts the crude rate of all outcomes by level of exposure to overcrowding in the pediatric emergency department.

The association between crowding in the pediatric emergency department and the odds of hospital admission within 7 days or mortality within 14 days of discharge from the index visit varied by acuity of the index visit and between sites. Nine of the 12 analyses (4 provinces $x 3$ acuity groups) yielded point estimates showing a positive association between crowding in the pediatric emergency department and hospital admission within 7 days or death within 14 days of discharge from the index visit. However, the magnitudes of the odds ratios (ORs) were relatively modest (from 1.02 to 1.03 per hour across provinces for high-acuity visits, from 0.99 to 1.02 per hour for medium-acuity visits, and from 0.98 to 1.11 per hour for low-acuity visits) and all of the $95 \% \mathrm{Cls}$ touched or crossed 1.0 (Figure 2). In further analysis using only hospital admission within 7 days as the outcome

Table 2: Outcomes of pediatric emergency department visits

\begin{tabular}{|c|c|c|c|c|}
\hline \multirow[b]{2}{*}{ Outcome } & \multicolumn{4}{|c|}{ No. $(\%)^{\star}$} \\
\hline & Ontario & Manitoba & Alberta & British Columbia \\
\hline No. discharged at index visits & 756034 & 207902 & 466479 & 176833 \\
\hline Index hospital admission & $74845(9.0)$ & $16895(7.5)$ & $41679(8.2)$ & $15717(8.2)$ \\
\hline Mortality within 14 days of ED discharge (per 100000 ) & $39(4.7)$ & $<6 \ddagger$ & $25(4.9)$ & $13(7.4)$ \\
\hline Return ED visit within 7 days of ED discharge & $75343(9.1)$ & $20992(10.1)$ & 43473 (8.6) & $15680(8.9)$ \\
\hline $\begin{array}{l}\text { Note: } E D=\text { emergency department. } \\
\text { *Unless stated otherwise. } \\
\text { IIncludes only the first visit within a 7-day period. } \\
\text { ‡No rates reported for low counts. }\end{array}$ & & & & \\
\hline
\end{tabular}


(i.e., excluding mortality), the results were nearly identical, likely because deaths were rare and contributed negligibly to the total number of composite outcomes.

Ten of the 12 analyses yielded point estimates showing a positive association between crowding in the pediatric emergency department and admission at the index visit. The magnitude of the ORs varied substantially across provinces, from 1.01 to 1.08 per hour in the high-acuity group to 0.99 to 1.04 per hour in the medium-acuity group (Figure 3). Similarly, 10 of the 12 analyses yielded point estimates showing a positive association between crowding in the pediatric emergency department and a return visit within 7 days of the index visit. The magnitude of these associations overall was also modest and significant only in the lowacuity group (Figure 4).
CTAS 1 or 2
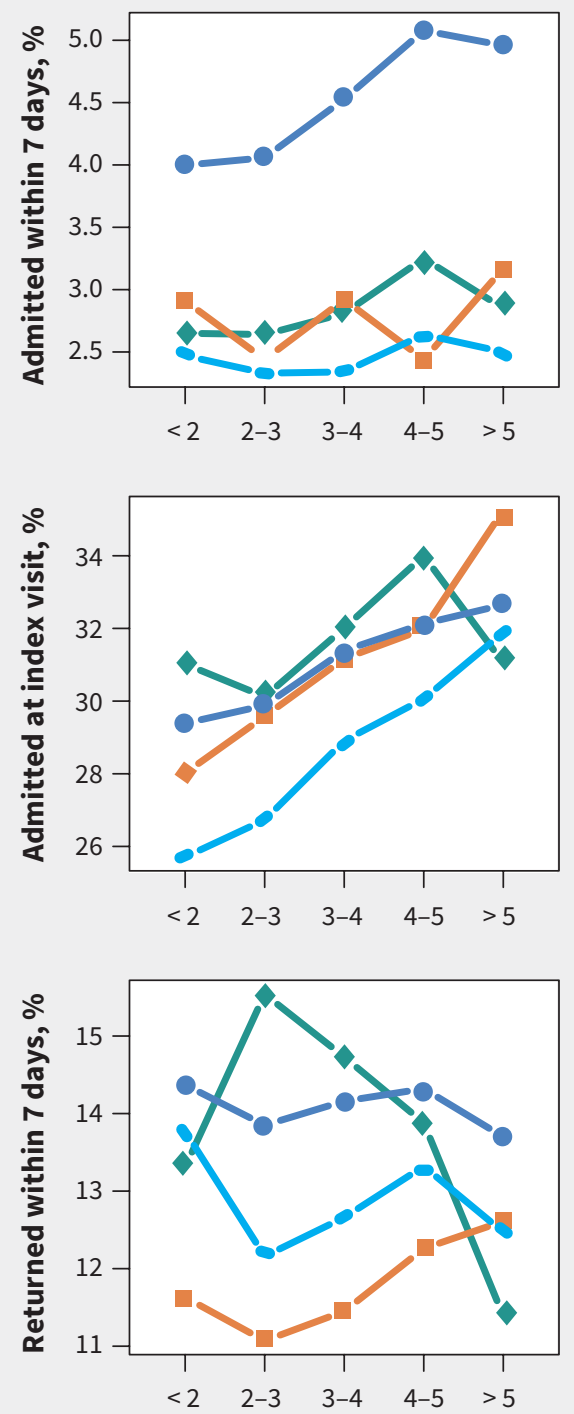

CTAS 3
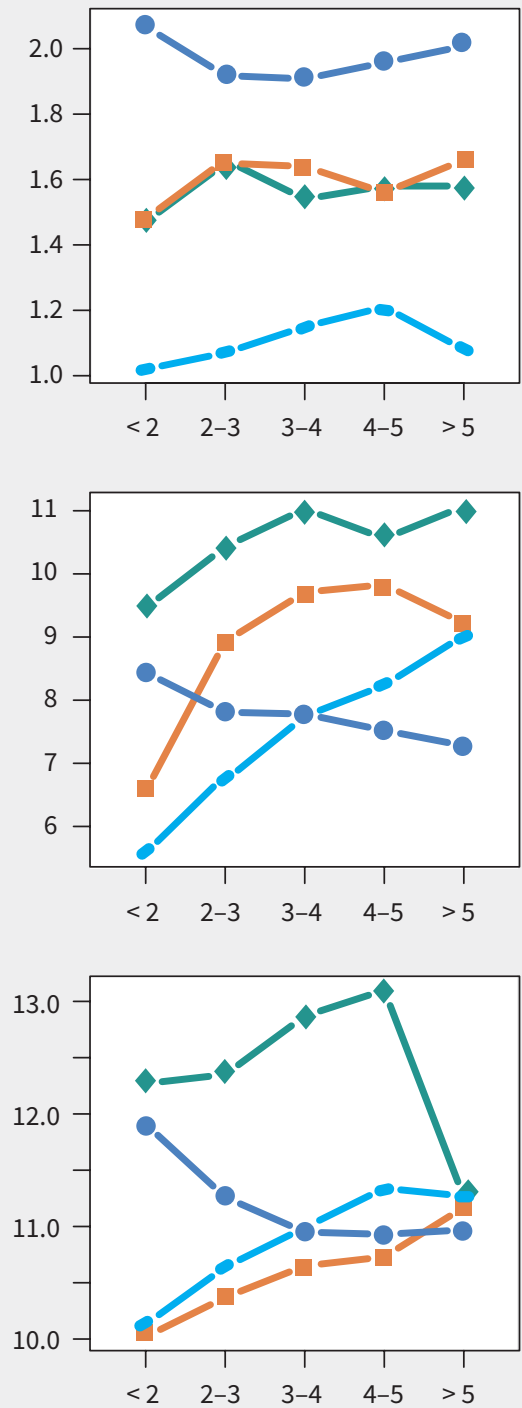

CTAS 4 or 5
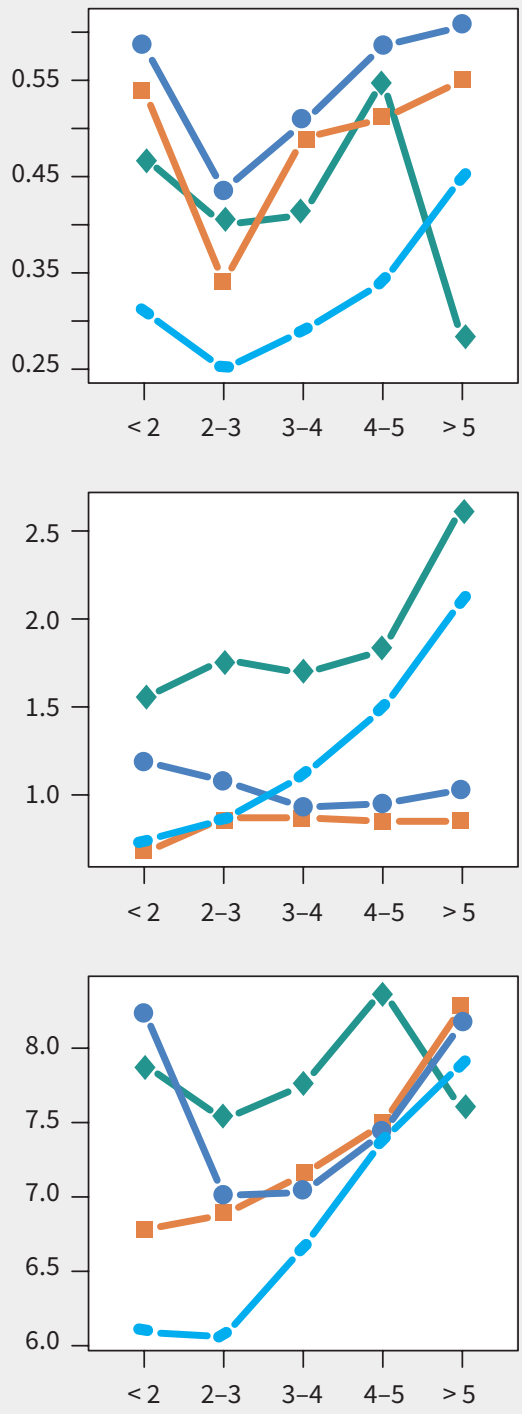

Departmental mean LOS in preceding 8 hours, hr 


\section{Interpretation}

In our multicentre cohort study of visits to the pediatric emergency department, we found no significant association between measures of crowding and either hospital admission within 7 days or mortality within 14 days after discharge. We did, however, observe an increase in the odds of hospital admission at the index visit with increasing departmental length of stay among the sickest children (CTAS score 1-3) and in return visits to the emergency department in the low-acuity group.

This study's main strength is its use of large data sets from multiple health regions in Canada. The multiprovincial data set provides an opportunity to explore the consistency, strength and variability of associations between emergency department crowding and adverse effects on measures of patient outcomes across different environments.

Studies in mixed or adult emergency departments have shown an association between crowding and increased mortality or excess hospital admission..$^{2,9,20-22}$ The most comparable previous studies are those of Guttmann and colleagues from Ontario and our group's earlier single-centre study in BC..$^{10,23}$ In their study of emergency department visits in a mostly adult sample (including children, but without separate analyses), Guttmann and colleagues observed ORs of 1.8 to 2.0 for hospital admission and mortality among patients discharged during shifts with a mean length of stay of 6 hours or longer compared with those with a

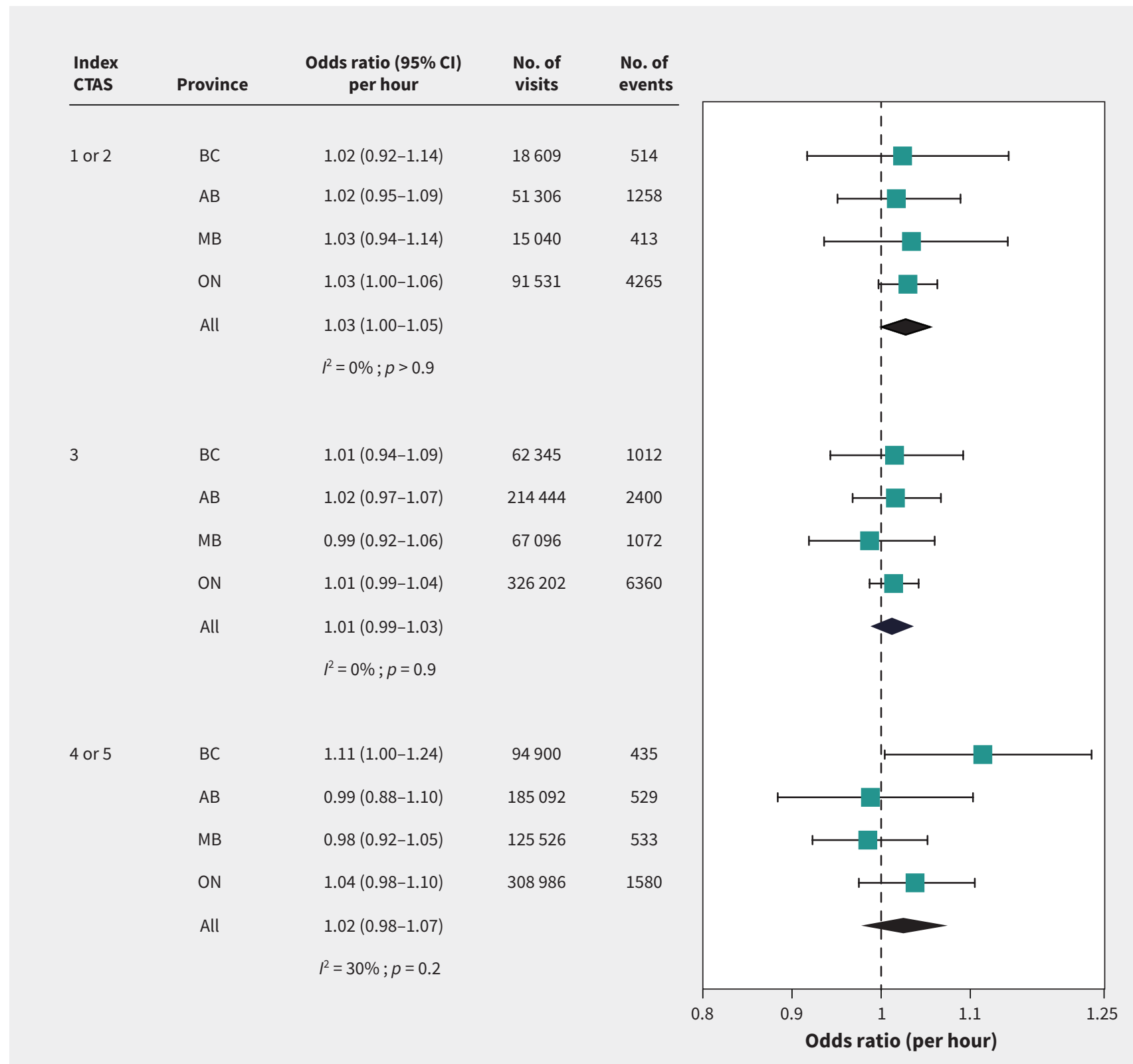

Figure 2: Odds of hospital admission within 7 days or mortality within 14 days of discharge from the pediatric emergency department, by triage acuity, with increasing departmental mean length of stay in the 8 hours before arrival. Note: $\mathrm{Cl}=$ confidence interval, CTAS = Canadian Triage and Acuity Scale. 
mean length of stay of less than 1 hour. ${ }^{10}$ The total sample of that study was larger than our individual provincial samples, and mortality in their mixed population study was 14 -fold higher than in our pediatric emergency department population (70/100000 v. $5 / 100000)$. Owing to the low mortality rate in children in general, our primary outcome was driven by hospital admission after discharge, which was also lower than that of the Ontario study, though more comparable (1.3\% v. $1.8 \%$ ), rather than mortality. In our group's prior single-centre pediatric emergency department study, we found an association between measures of crowding and odds of hospital admission at the index visit. ${ }^{23}$

The 2 earlier studies used a different measure of crowding that introduced the possibility of reverse causality. Although both used departmental mean length of stay, this was derived for the shift of the index patient's arrival, excluding that patient's own length of stay. The potential flaw in using this definition and the challenge it creates for interpreting causality relates to the timing of the index patient arrival within that shift and the resources required for that individual patient. For example, if index patient $A$ arrived at the beginning of an uncrowded shift and required substantial resources, the mean shift length of stay for all others arriving after patient A would increase, and some patients might leave without being seen. In this example, the measures of crowding assigned to patient $A$ (mean shift length of stay, left without being seen) are inflated by their own contribution to emergency department resource use. Moreover,

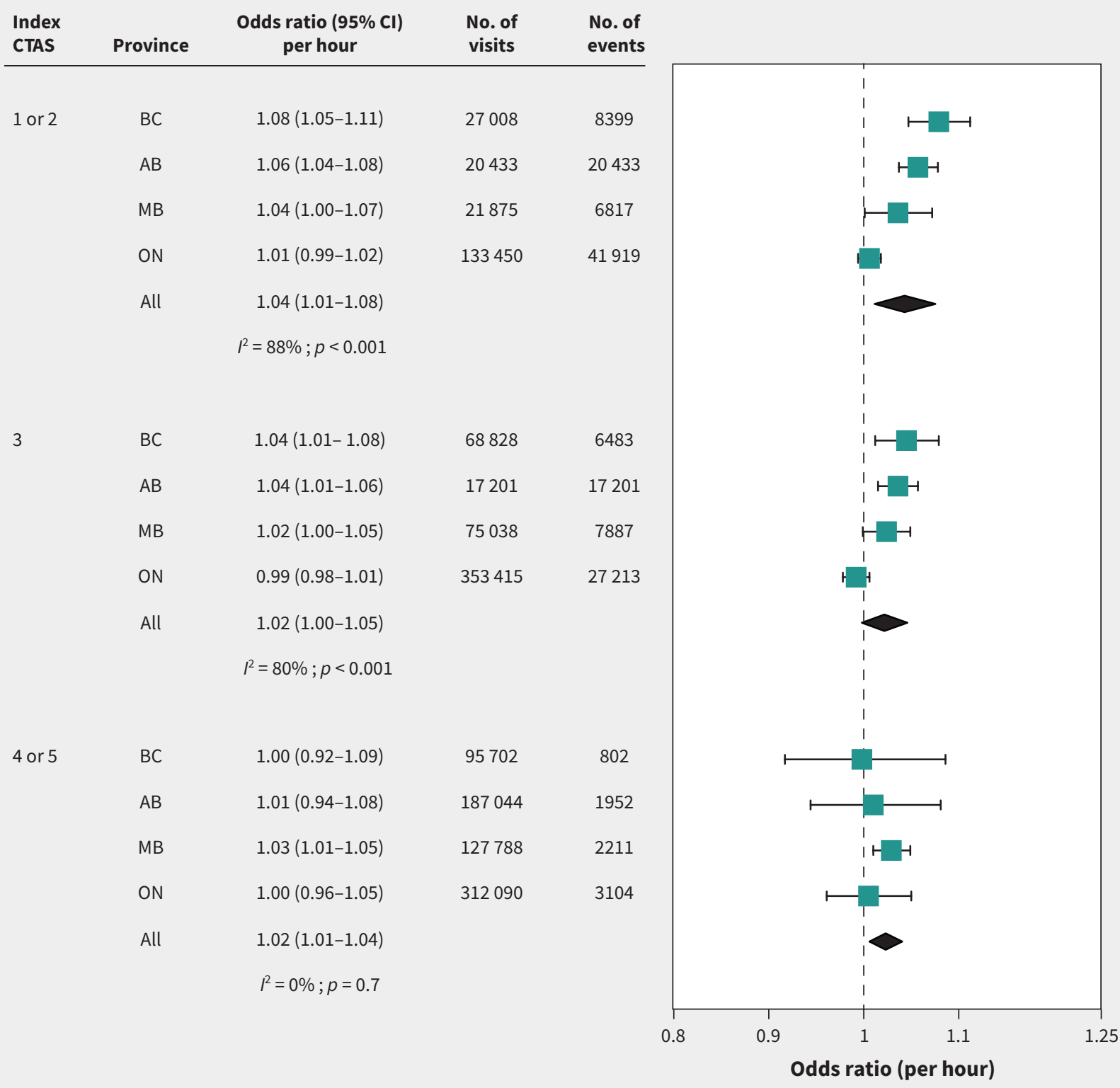

Figure 3: Odds of hospital admission at index visit to the pediatric emergency department with increasing departmental mean length of stay in the 8 hours before arrival. Note: $\mathrm{Cl}=$ confidence interval, CTAS = Canadian Triage and Acuity Scale. 
if patient A was eventually admitted to the intensive care unit (an adverse outcome), the observed positive association between exposure and outcome might represent reverse causality. To address this problem, our current study used mean length of stay of all patients in the pediatric emergency department for the 8 hours preceding the index visitor's arrival to ensure the exposure was measured in a consistent way for all index visits irrespective of arrival time and to mitigate the potential for reverse causality.

Whereas recent literature on pediatric emergency care describing the impact of emergency department crowding has focused largely on quality-of-care measures, ${ }^{13-15,24,25}$ our study adds new findings related to health care use. We observed an association between crowding in the pediatric emergency department and the odds of hospital admission at the index visit among high-acuity patients (CTAS- 1 and CTAS-2) across 3 of 4 provinces. Possible explanations include a delay in timely initiation of medical interventions that could lead to deterioration requiring hospital admission; alternatively, clinicians may respond to emergency department crowding with rising levels of caution in their disposition decision-making. Although the underlying cause of this association cannot be determined from this study, this association represents a previously undescribed impact of crowding on health care use with substantial cost implications.

\section{Return within 7 days}

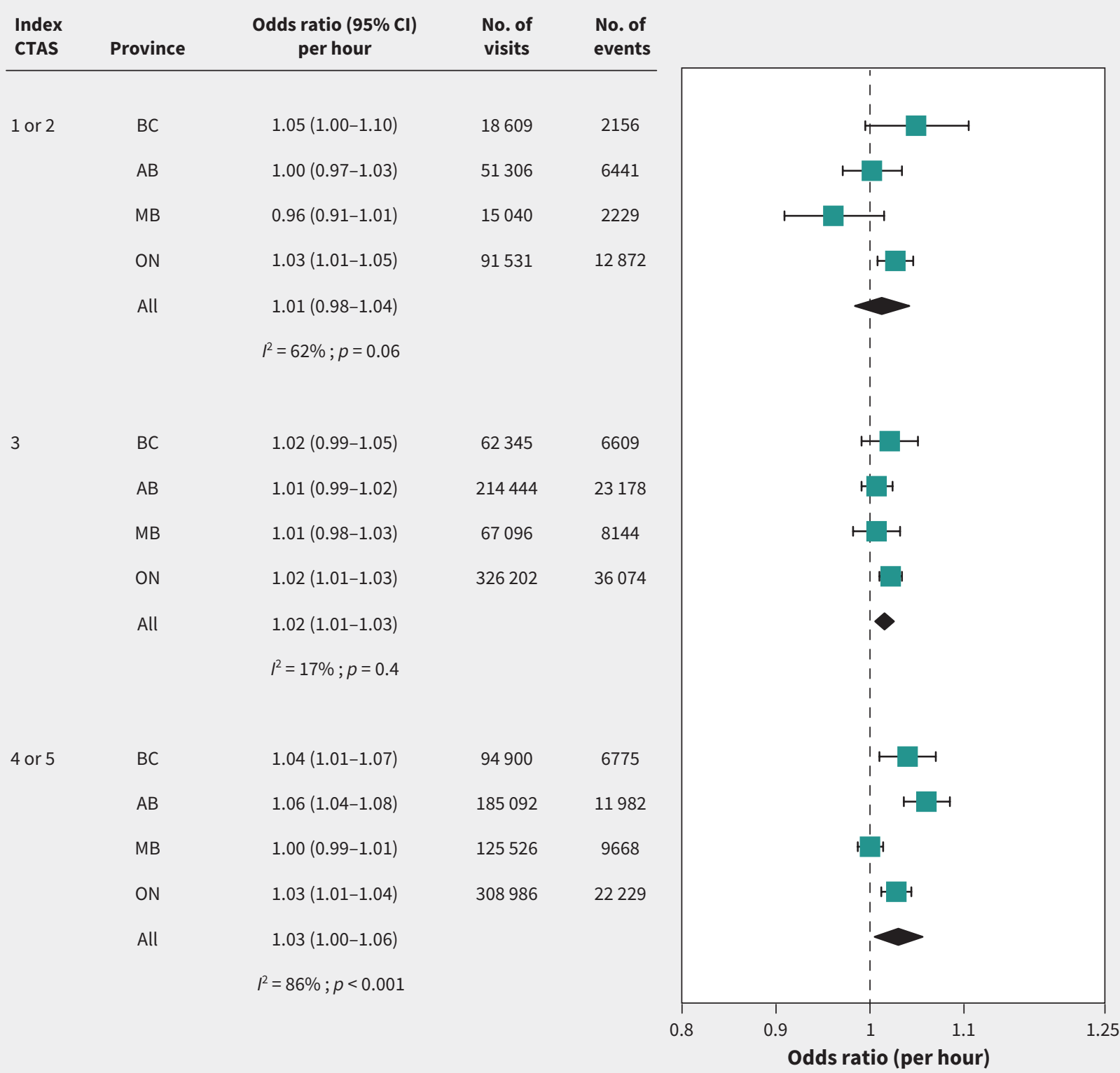

Figure 4: Odds of returning to the pediatric emergency department within 7 days of discharge with increasing departmental mean length of stay in the 8 hours before arrival. Note: $\mathrm{Cl}=$ confidence interval, CTAS = Canadian Triage and Acuity Scale. 
Finally, although the volume of emergency department visits increased annually across the 5-year study period, the overall length of stay remained stable. Potential explanations include operational and staffing adjustments during the study period. Some sites, for example, increased emergency department capacity and others enrolled in provincial fee-for-performance programs targeting length of stay or waiting time. In Alberta, a second emergency department staffed by clinicians from the pediatric emergency department opened in Calgary in 2013, resulting in additional staffing that may have affected the length of stay at the existing hospitals. In Ontario, 3 of the 4 sites participated in the Emergency Room Wait Time Strategy program (starting in 2009/10), ${ }^{26}$ which provided funding to increase emergency departments' capacity to meet specific length-of-stay benchmarks. The sites in BC and Manitoba were not involved in any provincial initiatives to increase emergency department capacity and had only minimal changes in staffing coverage during the study period.

\section{Limitations}

The main limitations of the study are the retrospective design and use of administrative databases, which depend on the accuracy of entered data and are limited in detail. Administrative databases do not capture other exposure variables that may confound the association between crowding and negative patient outcomes. For example, variability in emergency department clinicians' adherence to available evidence-based clinical guidelines, risk tolerance, clinical decision-making and efficiency may affect both emergency department crowding and patient outcomes. ${ }^{27-29}$ Another variable of interest, which we could not access for this study, was inpatient hospital occupancy rates at the time of emergency department index arrival. The impact of not including these variables as potential confounders is difficult to estimate, as no prior studies have reported on their association with emergency department length of stay and clinical outcome measures.

Although we report pooled estimates, differences in health care systems, populations served, and variations in practice and data elements across provinces raise concerns about whether the models estimate a common parameter across sites, and use of a single summary estimate of association may not be appropriate given this heterogeneity.

We acknowledge that the data we used are old and varied in availability across provinces; this reflects challenges and time required to obtain multiprovince data in light of specific privacy and ethics requirements at each site and health authority. However, limited epidemiologic studies have not shown a change in the rate of pediatric emergency department use or CTAS distribution (as a measure of acuity) in the last decade.

\section{Conclusion}

Although we did not find a significant association between crowding in the pediatric emergency department and hospital admission within 7 days or mortality within 14 days after discharge, we identified an association between hospital admission at index emergency department visit among high-acuity visits, as well as return visits within 7 days among low-acuity visits with increasing emergency department crowding. Prior reports of the association between emergency department crowding and poor patient outcomes have been limited to the adult population. Our observed associations suggest that crowding in the pediatric emergency department has implications for use of health services.

\section{References}

1. Affleck A, Parks P, Drummond A, et al. Emergency department overcrowding and access block. CJEM 2013;15:359-84.

2. Richardson DB. Increase in patient mortality at 10 days associated with emergency department overcrowding. Med J Aust 2006;184:213-6.

3. Rondeau KV, Francescutti LH. Emergency department overcrowding: the impact of resource scarcity on physician job satisfaction. J Healthc Manag 2005; 50:327-40.

4. Hoot NR, Aronsky D. Systematic review of emergency department crowding: causes, effects, and solutions. Ann Emerg Med 2008;52:126-36.

5. Bernstein SL, Aronsky D, Duseja R, et al. The effect of emergency department crowding on clinically oriented outcomes. Acad Emerg Med 2009;16:1-10.

6. Bond K, Ospina MB, Blitz S, et al. Frequency, determinants and impact of overcrowding in emergency departments in Canada: a national survey. Healthc $Q$ 2007;10:32-40

7. Schull MJ, Lazier K, Vermeulen M, et al. Emergency department contributors to ambulance diversion: a quantitative analysis. Ann Emerg Med 2003;41:467-76.

8. Pines JM, Hollander JE, Localio AR, et al. The association between emergency department crowding and hospital performance on antibiotic timing for pneumonia and percutaneous intervention for myocardial infarction. Acad Emerg Med 2006;13:873-8.

9. Sun BC, Hsia RY, Weiss RE, et al. Effect of emergency department crowding on outcomes of admitted patients. Ann Emerg Med 2013;61:605-11.e6.

10. Guttmann A, Schull MJ, Vermeulen MJ, et al. Association between waiting times and short- term mortality and hospital admission after departure from emergency department: population based cohort study from Ontario, Canada. BMJ 2011;342:d2983.

11. Stang AS, McGillivray D, Bhatt M, et al. Markers of overcrowding in a pediatric emergency department. Acad Emerg Med 2010;17:151-6.

12. Doan $\mathrm{Q}$, Genuis ED, Yu A. Trends in use in a Canadian pediatric emergency department. CJEM 2014;16:405-10.

13. Sills MR, Fairclough D, Ranade D, et al. Emergency department crowding is associated with decreased quality of care for children with acute asthma. Ann Emerg Med 2011;57:191-200.e1-7.

14. Kennebeck SS, Timm NL, Kurowski EM, et al. The association of emergency department crowding and time to antibiotics in febrile neonates. Acad Emerg Med 2011;18:1380-5

15. Shenoi R, Ma L, Syblik D, et al. Emergency department crowding and analgesic delay in pediatric sickle cell pain crises. Pediatr Emerg Care 2011;27:911-7.

16. Gouin S, Gravel J, Amre DK, et al. Evaluation of the Paediatric Canadian Triage and Acuity Scale in a pediatric ED. Am J Emerg Med 2005;23:243-7.

17. Timm NL, Ho ML, Luria JW. Pediatric emergency department overcrowding and impact on patient flow outcomes. Acad Emerg Med 2008;15:832-7.

18. Hwang U, Mccarthy ML, Aronsky D, et al. Measures of crowding in the emergency department: a systematic review. Acad Emerg Med 2011;18:527-38.

19. Mapelli E, Black T, Doan Q. Trends in pediatric emergency department utilization for mental health-related visits. J Pediatr 2015;164:905-10.

20. Sprivulis PC, Da Silva J-A, Jacobs IG, et al. The association between hospital overcrowding and mortality among patients admitted via Western Australian emergency departments. Med J Aust 2006;184:208-12.

21. Miró O, Antonio MT, Jiménez S, et al. Decreased health care quality associated with emergency department overcrowding. Eur J Emerg Med 1999;6:105-7. 
22. Pines JM, Pollack CV, Diercks DB, et al. The association between emergency department crowding and adverse cardiovascular outcomes in patients with chest pain. Acad Emerg Med 2009;16:617-25.

23. Chan M, Meckler G, Doan Q. Paediatric emergency department overcrowding and adverse patient outcomes. Paediatr Child Health 2017;22:377-381.

24. Sills MR, Fairclough D, Ranade D, et al. Emergency department crowding is associated with decreased quality of care for children. Pediatr Emerg Care 2011;27:837-45.

25. Depinet HE, lyer SB, Hornung R, et al. The effect of emergency department crowding on reassessment of children with critically abnormal vital signs. Acad Emerg Med 2014;21:1116-20.
26. Ontario Ministry of Health and Long-Term Care. Ontario wait times. Available: www.health.gov.on.ca/en/pro/programs/waittimes/surgery/strategy.aspx (accessed 2019 May 16).

27. Jain S, Elon LK, Johnson B, et al. Physician practice variation in the pediatric emergency department and its impact on resource use and quality of care. Pediatr Emerg Care 2010;26:902-8.

28. Jain S, Frank G, McCormick K, et al. Impact of physician scorecards on emergency department resource use, quality, and efficiency. Pediatrics 2015;136:e670-9.

29. Gaucher N, Bailey B, Gravel J. Impact of physicians' characteristics on the risk of admission among children visiting a pediatric emergency department. Pediatr Emerg Care 2012;28:120-4.
Competing interests: Quynh Doan reports a Scholars Award from the Michael Smith Foundation for Health Research during the conduct of the study. No other competing interests were declared.

This article has been peer reviewed.

Affiliations: Division of Pediatric Emergency Medicine, Department of Pediatrics (Doan, Meckler), University of British Columbia; School of Population and Public Health (Wong), University of British Columbia; BC Children's Hospital Research Institute (Doan, Meckler), Vancouver, BC; Alberta Children's Hospital Research Institute (Johnson, Stang), University of Calgary, Calgary, Alta; Stollery Children's Hospital, and Women and Children's Health Research Institute (Dixon), University of Alberta, Edmonton, Alta.; Pediatric Emergency Medicine (Sawyer), University of Manitoba; Children's Hospital, Health Sciences Centre Winnipeg (Sawyer), Winnipeg, Man.; Paediatric Emergency Medicine (Principi), University of Toronto; The Hospital for Sick Children (Principi), Toronto, Ont.; Department of Pediatrics (Kam), McMaster University; McMaster Children's Hospital (Kam), Hamilton, Ont.; Paediatric Emergency Medicine (Joubert), Western University; Children's Hospital of Western Ontario (Joubert), London, Ont.; Département de pédiatrie (Gravel), Université de Montréal; CHU SainteJustine (Gravel), Montréal, Que.; Department of Pediatrics (Jabbour), University of Ottawa;
Children's Hospital of Eastern Ontario (Jabbour), Ottawa, Ont.; ICES (Guttmann); Department of Paediatrics (Guttmann), University of Toronto, Toronto, Ont.

Contributors: All authors contributed to the study design (exposure and outcome measure conceptualization). Quynh Doan, Antonia Stang, Andrew Dixon, Scott Sawyer, Tania Principi, April Kam and Astrid Guttmann contributed to the data acquisition. Hubert Wong performed the data analysis. Quynh Doan, Hubert Wong, Garth Meckler, David Johnson, Antonia Stang, Andrew Dixon, Scott Sawyer, Tania Principi, Gary Joubert, Jocelyn Gravel, Mona Jabbour and Astrid Guttmann contributed to the data interpretation. Quynh Doan drafted the manuscript, which all authors revised. All of the authors approved the final version to be published and agreed to be accountable for all aspects of the work.

Funding: This project was supported by an establishment award provided to Quynh Doan by the BC Children's Hospital Research Institute.

Data sharing: None of the data used in this study can be shared by the investigators. However, access to the Ontario data may be requested from ICES by other researchers by following the procedures detailed at www. ices.on.ca/DAS/Submitting-your-request.

Acknowledgements: This study made use of de-identified data from the ICES Data Repository, which is managed by ICES with support from its funders and partners: Canada's Strategy for Patient-Oriented Research (SPOR), the Ontario SPOR SUPPORT (Support for People and Patient-Oriented Research and Trials) Unit, the Canadian Institutes of Health Research and the Government of Ontario. The opinions, results and conclusions reported are those of the authors. No endorsement by ICES or any of its funders or partners is intended or should be inferred. Parts of this material are based on data and information compiled and provided by the Canadian Institute for Health Information (CIHI). However, the analyses, conclusions, opinions and statements expressed herein are those of the authors, and not necessarily those of $\mathrm{CIHI}$. The authors would also like to acknowledge the contribution of the data analysts and custodians from all 4 provinces as outlined in Appendix 2.

Disclaimer: This study was supported by ICES, which is funded by an annual grant from the Ontario Ministry of Health and Long-Term Care (MOHLTC). The opinions, results and conclusions reported in this paper are those of the authors and are independent from the funding sources. No endorsement by ICES or the Ontario MOHLTC is intended or should be inferred.

Accepted: Apr. 26, 2019

Correspondence to: Quynh Doan, qdoan@bcchr.ca 